\title{
Sleep Quality and Restless Legs Syndrome in Patients with Ankylosing Spondylitis
}

\author{
(D) Gülbün Asuman Yüksel ${ }^{1}$, (i) Duygu Kurtuluş², (1) Hülya Tireli ${ }^{1}$ \\ ${ }^{1}$ Department of Neurology, Haydarpasa Numune Training and Research Hospital, Istanbul, Turkey \\ ${ }^{2}$ Department of Physical Medicine and Rehabilitation, Umraniye Training and Research Hospital, Istanbul, Turkey
}

\begin{abstract}
Introduction: We aimed to investigate restless leg syndrome (RLS) prevalence and sleep quality in patients with ankylosing spondylitis (AS) and to explore the association between RLS and measures of disease activity and physical function in AS. Methods: Twenty (5 female, 15 male) consecutive patients with AS and 20 ( 5 female, 15 male) healthy volunteers were enrolled in this study. RLS was diagnosed by International Restless Legs Syndrome Study Group (IRLSSG-2003). Sleep quality was evaluated using the Pittsburgh Sleep Quality Index (PSQI), where scores $>5$ indicated poor sleep quality.

Results: Prevalence of RLS was 55\% (11 patients) in patients with AS. The mean RLS disability value was 21 (SD, 7.9). The modified Schober test levels were significantly higher $(\mathrm{p}=0.011)$ and Bath Ankylosing Spondylitis Functional Index (BASFI) scores were significantly lower $(p=0.016)$ in the RLS group than in the non-RLS group. The Bath AS Disease Activity Index (BASDAI) scores were lower in the RLS group than in the non-RLS group, but the difference was not statistically significant $(p=0.16)$. Sleep quality was poor in 12 patients [PSQI score=7.09 (SD, 3.67)].

Discussion and Conclusion: We emphasized that it is important to determine the existence of RLS and poor sleep quality in patients with AS. However, further examination by applying the diagnostic criteria for RLS and PSQI in more number of patients with AS will be useful.

Keywords: Ankylosing spondylitis; restless legs syndrome; sleep quality.
\end{abstract}

A nkylosing Spondylitis (AS) is a chronic inflammatory rheumatic disease that primarily affects the spine and sacroiliac and peripheral joints. Its main symptoms include pain and stiffness ${ }^{[1,2]}$.

Restless Legs Syndrome (RLS) is a chronic neurological condition associated with significant sleep disturbances and a resulting considerable negative impact on the patients' quality of life ${ }^{[3]}$. It is characterized by an irresistible urge to move, usually accompanied or caused by uncomfortable sensations in the legs ${ }^{[4]}$. These sensations generally occur while the patient is at rest and worsen in the evening or at night and can be temporarily relieved by movement $[3$,
5]. RLS is one of the leading causes of insomnia, affecting more than $5 \%-10 \%$ of the population ${ }^{[6-9]}$. The incidence in women is twice than that in men ${ }^{[6,9]}$. RLS is associated with a variety of conditions, including iron deficiency, uremic neuropathy, alcohol use, diabetes, amyloid, and barbiturate and phenothiazine therapies ${ }^{[3,9-12]}$. The pathophysiology of RLS remains unclear. However, pharmacological evidence indicates that the disorder is related to dopaminergic dysfunction in the central nervous system ${ }^{[3,11]}$.

The diagnosis of RLS is exclusively based on the four criteria proposed by the International Restless Legs Syndrome Study Group (IRLSSG-2003) ${ }^{[13]}$. IRLSSG developed a rating

Correspondence (İletişim): Gülbün Asuman Yüksel, M.D. Haydarpaşa Numune Eğitim ve Araştırma Hastanesi, Nöroloji Anabilim Dalı, Istanbul, Turkey Phone (Telefon): +90 5053983063 E-mail (E-posta): gulbunyuksel@yahoo.com 
scale for measuring the severity of RLS [International Restless Legs Scale (IRLS)] based on the questions proposed by its members who possessed clinical expertise regarding RLS [14]. Rheumatological diseases, including rheumatoid arthritis (RA), fibromyalgia (FM), and Sjögren syndrome, have also been reported to be associated with RLS symptoms in a large percentage of patients with these disorders $[3,7]$. However, we could not find data on the prevalence of RLS symptoms in patients with AS.

Therefore, this study was carried out with the following aims:

1. To investigate the prevalence of RLS in patients with AS

2. To explore the associations between RLS and measures of disease activity and physical function in AS.

\section{Materials and Methods}

Twenty consecutive patients ( 15 male, 5 female) with AS defined according to the modified New York criteria 1984 were enrolled in the study ${ }^{[15]}$. The mean age of the patients was 39.6 years (SD, 11.3; range, $21-56$ years). The control group consisted of 20 (15 male, 5 female) healthy volunteers with a mean age of 39.05 years (SD, 10.6; range, 21-59 years). The disease duration and current treatment were determined for all patients. The modified Schober test, chest expansion, tragus to wall distance, and right and left lateral flexion measures of the spine were assessed. Neurological examination and detailed laboratory investigation [complete blood count, biochemical parameters, C-reactive protein (CRP) levels, erythrocyte sedimentation rate (ESR), and ferritin levels)] were performed in all patients and controls. The patients were interrogated for myalgia, venous stasis, leg edema, arthritis, leg cramps, positional discomfort, and habitual foot tapping. The conditions associated with RLS include neuropathy, radiculopathy, iron deficiency anemia, uremic conditions, thyroid disease, and Parkinson disease. Patients with metabolic abnormalities, systemic disease, and low ferritin levels were not included in the study. RLS can also be observed in patients with polyneuropathy. Hence, nerve conduction study was performed in all patients for excluding the existence of polyneuropathy.

Disease activity and functional status of patients were evaluated using the Bath Ankylosing Spondylitis Disease Activity Index (BASDAI) and Bath Ankylosing Spondylitis Functional Index (BASFI), respectively $[16,17]$. Pain and patient global assessments were determined with the visual analog scale (VAS; grades 0-10), Multi-Dimensional Health Assessment Questionnaire (MDHAQ; grades 0-10), and MDHAQ-Routine Assessment of Patient Index Data [MDHAQ-RAPID-3; including physical function (grades $0-10)$ ], pain (0-10), and patient global estimate $(0-10)$ (grades $0-30){ }^{[18]}$.
The diagnosis of RLS was exclusively based on the four criteria and supportive clinical features proposed by IRLSSG-2003 [13]. The diagnosis was made by clinical evaluation and some questionnaires assessed by two neurologists.

IRLS was administrated to assess RLS severity ${ }^{[14]}$. Sleep disturbance was evaluated using the Pittsburgh Sleep Quality Index (PSQI) ${ }^{[19]}$.

Diagnostic criteria for RLS are shown in Table 1. Objective measures of RLS severity were evaluated using IRLS, which consists of 10 items that are graded from 0 to 4; a total score is then generated and ranges from 0 to 40 . The items used to calculate the total IRLS score can be grouped as follows: factors related to symptoms severity (IRLS symptoms subscale) and those related to the impact of IRLS on daily living or quality of life (IRLS symptoms impact subscale). Scores of 0-10 indicate mild RLS severity, 11-20 indicate moderate, 21-30 indicate severe, and 31-40 indicate very severe ${ }^{[14]}$.

PSQI is a self-reporting measure assessing sleep quality and disturbances in the previous month. It includes 19 items, generating seven component scores: subjective sleep quality, sleep latency, sleep duration, habitual sleep efficiency, sleep disturbances, use of sleeping medication, and daytime dysfunction. Each item is scored between 0 and 3 points according to the frequency of symptoms. A global score can range from 0 (no sleep difficulty) to 21 (severe sleep difficulty). A total score of $\geq 5$ is considered suggestive of clinically poor sleep quality ${ }^{[19]}$. Excessive daytime sleepiness is the overwhelming urge to sleep during the day when one would rather be active. Epworth Sleepiness Scale (ESS) was used to assess excessive daytime sleepi-

Table 1. Essential diagnostic criteria of the International RLS Study Group

\section{Diagnostic criteria}

1. An urge to move the legs, usually accompanied or caused by uncomfortable and unpleasant sensations in the legs

2. The urge to move or unpleasant sensations begin or worsen during periods of rest or inactivity, such as lying or sitting

3. The urge to move or unpleasant sensations are partially or totally relieved by movement, such as walking or stretching, at least as long as the activity continues

4. The urge to move or unpleasant sensations are worse in the evening or night than during the day or only occur in the evening or night

\section{Supportive clinical features}

1. Sleep disturbance

2. Periodic leg movements

3. Response to dopaminergic therapy

4. Family history

5. Normal medical/physical evaluation 
ness. It consists of 8 items, each graded from 0 to 3 , and the total score can range from 0 to 24 . A total score of $\geq 10$ is indicative of excessive daytime sleepiness ${ }^{[20]}$. The degree of fatigue was evaluated using the Fatigue Severity Scale (FSS), which consists of 9 items, each graded from 0 to 7 . The total score can range from 0 to 63 , where scores $\geq 36$ indicate a high degree of fatigue ${ }^{[21]}$.

The necessary approval by the local institutional review board was obtained for this scientific research.

\section{Statistical Analysis}

Statistical Package for Social Sciences (SPSS) for Windows 16.0 was used for statistical analysis. One sample t-test, independent t-test, and F-test were used for quantitative comparisons. The Pearson's chi-square and Fischer's exact tests were used for the comparison of categorical variables. The results were evaluated within 95\% confidence interval, and $p<0.05$ was considered statistically significant. For continuous variables (assumed normal distribution), the Pearson's correlation coefficient was used. The Student's t-test was used to compare the mean values of electrophysiological tests between the patients and controls. The data were analyzed using SPSS II computer program, version 10 . The data were expressed as mean $\pm 2 S D$.

\section{Results}

The demographic, clinical, and laboratory characteristics of patients with AS are shown in Table 2. RLS was found in 11 patients with AS (55\%) and in one healthy control (5\%). The mean RLS disability value was 21 (SD, 7.91) in patients with AS. Among AS patients with RLS, two (18.1\%) had very severe RLS (score, 31-40), three (27.2\%) had severe RLS (21$30)$, five $(45.4 \%)$ had moderate RLS (11-20), and one (9.0\%)
Table 2. Demographic details, spinal mobility, and disease-related characteristics of patients with AS

Age at presentation, mean $\pm \mathrm{SD}$ (years)

$39.6 \pm 11.3$

Sex, number (male/female)

$15 / 5$

Age at onset, mean \pm SD (years)

$31.8 \pm 9$

Disease duration, mean \pm SD (years)

$7.8 \pm 6.6$

BASDAI, mean $\pm S D$

$5.2 \pm 2.2$

BASFI, mean \pm SD

$4.2 \pm 2.5$

MDHAQ- function, mean \pm SD

$2.4 \pm 2.2$

Pain, mean \pm SD

$5 \pm 2.9$

Patient-global, mean $\pm S D$

$4.9 \pm 2.4$

MDHAQ -RAPID-3, mean \pm SD

$12.4 \pm 6$

Physician-global, mean \pm SD

$4.6 \pm 2.5$

Modified Schober test, mean \pm SD $(\mathrm{cm})$

$4.2 \pm 2.9$

Lateral flexion-right, mean $\pm \mathrm{SD}(\mathrm{cm})$

$10.7 \pm 7.3$

Lateral flexion-left, mean \pm SD $(\mathrm{cm})$

$11 \pm 7.5$

Tragus to wall distance, mean $\pm S D(\mathrm{~cm})$

$20.9 \pm 5.8$

Chest expansion, mean \pm SD $(\mathrm{cm})$

$3.9 \pm 2.2$

Erythrocyte sedimentation rate, $\mathrm{mm} / \mathrm{h}$

$14 \pm 14.9$

C-Reactive protein, $\mathrm{mg} / \mathrm{dl}$

$1.2 \pm 0.8$

BASDAI: Bath Ankylosing Spondylitis Disease Activity Index; BASFI: Bath Ankylosing Spondylitis Functional Index; MDHAQ: Multi-Dimensional Health Assessment Questionnaire; MDHAQ-RAPID-3: Multi-Dimensional Health Assessment Questionnaire-Routine Assessment of Patient Index Data.

had mild RLS (1-10). The disease duration was longer in the non-RLS group, but it was not statistically significant $(p=0.25)$. There was no significant correlation in terms of age, age at onset, and disease duration between the RLS and non-RLS groups. There were no significant differences in terms of ferritin levels between the RLS and non-RLS groups $(\mathrm{p}=0.86)$.

The mean PSQI was 7.09 (SD, 3.67; range, $3-14$ ), and $80 \%$ of patients had poor sleep quality. Higher PSQI levels were associated with worse sleep quality. There were no significant

Table 3. Association between PSQI and clinical disease scores

\begin{tabular}{lccc}
\hline & PSQI $\geq \mathbf{5}$ & PSQI $<\mathbf{5}$ & p/t \\
\hline Modified Schober $(\mathrm{cm})$ & $4.44 \pm 2.9$ & $2.88 \pm 2.8$ & $\mathrm{p}=0.335 / \mathrm{t}=0.991$ \\
Lateral flexion-right $(\mathrm{cm})$ & $11.06 \pm 7.5$ & $9.50 \pm 7.3$ & $\mathrm{p}=0.713 / \mathrm{t}=0.374$ \\
Lateral flexion-left $(\mathrm{cm})$ & $11.66 \pm 8$ & $8.75 \pm 5.7$ & $\mathrm{p}=0.507 / \mathrm{t}=0.677$ \\
Tragus to wall distance $(\mathrm{cm})$ & $20.09 \pm 5.5$ & $24 \pm 7$ & $\mathrm{p}=0.242 / \mathrm{t}=1.210$ \\
Chest expansion $(\mathrm{cm})$ & $3.69 \pm 1.8$ & $4.75 \pm 3.6$ & $\mathrm{p}=0.407 / \mathrm{t}=0.849$ \\
BASDAI clinical score $(0-10)$ & $5.43 \pm 2.1$ & $\mathrm{p}=0.328 / \mathrm{t}=1.050$ \\
BASFI clinical score $(0-10)$ & $4.47 \pm 2.3$ & $\mathrm{p}=0.348 / \mathrm{t}=0.964$ \\
MDHAQ function $(0-10)$ & $2.38 \pm 2.4$ & $3.13 \pm 3.3$ & $\mathrm{p}=0.969 / \mathrm{t}=0.040$ \\
Pain VAS (0-10) & $5.36 \pm 3.2$ & $2.43 \pm 1.6$ & $\mathrm{p}=0.388 / \mathrm{t}=0.884$ \\
Pt-global VAS (0-10) & $5.06 \pm 2.6$ & $3.88 \pm 1.6$ & $\mathrm{p}=0.686 / \mathrm{t}=0.411$ \\
MDHAQ-RAPID-3 (0-10) & $12.8 \pm 7.5$ & $4.5 \pm 1.1$ & $\mathrm{p}=0.612 / \mathrm{t}=0.616$ \\
\hline
\end{tabular}

BASDAI: Bath Ankylosing Spondylitis Disease Activity Index; BASFI: Bath Ankylosing Spondylitis Functional Index; MDHAQ: Multi-Dimensional Health Assessment Questionnaire; MDHAQ-RAPID-3: Multi-Dimensional Health Assessment Questionnaire-Routine Assessment of Patient Index Data; Pt-global: Patient global assessment. 
differences for clinical scores between patients with poor sleep quality and those with better sleep quality (Table 3). PSQI levels were higher in the RLS group than in the nonRLS group ( $8.4 \pm 3.6$ and $7.3 \pm 4$, respectively), but the difference was not statistically significant $(p=0.547)$.

Sleep quality and fatigue scores were evaluated. The PSQI, ESS, and FSS scores of patients with RLS were 7.09 \pm 3.67 , $8.35 \pm 6.28$, and $42.30 \pm 14.70$, respectively. The patients complained of worse sleep quality (80\%), excessive daytime sleepiness (45\%), and increased fatigue (80\%) (Table 4).

Fatigue scores were significantly higher $(p=0.039)$ and daytime sleepiness was increased in patients with worse sleep quality (Table 5).

The modified Schober test scores were significantly higher $(p=0.011)$ and BASFI scores were significantly lower $(p=0.016)$ in the RLS group than in the non-RLS group. There was a positive correlation between modified Schober and IRLS scores (57.6\%; $p=0.008)$. BASDAI scores were low in the RLS group, but they were not statistically significant $(p=0.16)$. No significant differences were found in other parameters between the RLS and non-RLS groups (Table 6). There were no significant differences in ESR and CRP levels between the RLS and non-RLS groups.

\section{Discussion}

RLS prevalence in the general population is estimated to be $2.5 \%-10 \%[3,7,10]$. In a study that examined RLS in patients with rheumatological conditions, $25 \%$ of patients with RA, 31\% with FM, 24\% with Sjögren syndrome, and $22 \%$ with scleroderma had RLS ${ }^{[3]}$. Salih et al. ${ }^{[10]}$ found RLS in $25 \%$ of patients with RA, and most were female (83\%). The prevalence of both RLS and rheumatological diseases increases with aging; therefore, these conditions may occur concomitantly. It is likely that many rheumatologists have encountered patients with RLS. In addition, RLS is often unrecognized, and rheumatological or painful disorders are suggested as the differential diagnosis, leading to referral to a rheumatologist ${ }^{[3]}$.

Table 4. Scores of sleep quality, daytime sleepiness, and fatigue in AS patients

\begin{tabular}{lcc}
\hline & $\mathbf{n},(\%)$ & Scores \\
\hline PSQI & 20 (total) & $7.09 \pm 3.67$ \\
$\quad \geq 5$ & $16(80)$ & $9.06 \pm 3.13$ \\
$\quad<5$ & $4(20)$ & $3.25 \pm 0.50$ \\
$(\geq 5:$ worse sleep quality) & & \\
ESS & 20 (total) & $8.35 \pm 6.28$ \\
$\quad \geq 10$ & $9(45)$ & $14.22 \pm 3.38$ \\
$\quad<10$ & $11(55)$ & $3.55 \pm 3.05$ \\
$(\geq 10:$ excessive daytime sleepiness) & & \\
FSS & 20 (total) & $42.30 \pm 14.70$ \\
$\quad \geq 36$ & $16(80)$ & $48.18 \pm 7.91$ \\
$\quad<36$ & $4(20)$ & $18.75 \pm 11.44$ \\
( $\geq 36:$ increased fatigue) & &
\end{tabular}

PSQI: Pittsburgh Sleep Quality Index; ESS: Epworth Sleepiness Scale; FSS:Fatigue Severity Scale.

Table 5. Association between PSQI scores and ESS and FSS scores

\begin{tabular}{lccc}
\hline & $\begin{array}{c}\text { PSQI } \geq \mathbf{5}(\mathbf{n}=\mathbf{1 6}) \\
\text { (Worse sleep quality) }\end{array}$ & $\begin{array}{c}\text { PSQI }<\mathbf{5}(\mathbf{n}=\mathbf{4}) \\
\text { (Better sleep quality) }\end{array}$ & p \\
\hline ESS & $9.50 \pm 6.38$ & $3.75 \pm 3.30$ & 0.102 \\
FSS & $45.63 \pm 12.92$ & $29.00 \pm 15.43$ & $0.039^{*}$
\end{tabular}

PSQI: Pittsburgh Sleep Quality Index; ESS: Epworth Sleepiness Scale; FSS: Fatigue Severity Scale.

Table 6. Comparison of clinical disease scores between the RLS and non-RLS groups

\begin{tabular}{lccc}
\hline & RLS group & Non-RLS group & p/t \\
\hline Modified Schober $(\mathrm{cm})$ & $5.50 \pm 2.8$ & $2.44 \pm 1.8$ & $\mathrm{p}=0.011^{*}, \mathrm{t}=2.818$ \\
Lateral flexion-right $(\mathrm{cm})$ & $12.55 \pm 7.1$ & $8.56 \pm 7.3$ & $\mathrm{p}=0.234, \mathrm{t}=1.232$ \\
Lateral flexion-left $(\mathrm{cm})$ & $14.05 \pm 7.4$ & $7.44 \pm 6.3$ & $\mathrm{p}=0.049^{*}, \mathrm{t}=2.11$ \\
Tragus to wall distance $(\mathrm{cm})$ & $19.45 \pm 4.2$ & $22.61 \pm 7.2$ & $\mathrm{p}=0.239, \mathrm{t}=1.217$ \\
Chest expansion $(\mathrm{cm})$ & $4.18 \pm 2.5$ & $3.56 \pm 1.9$ & $\mathrm{p}=0.545, \mathrm{t}=0.617$ \\
BASDAI clinical score (0-10) & $4.55 \pm 2.2$ & $5.96 \pm 2.05$ & $\mathrm{p}=0.160, \mathrm{t}=1.464$ \\
BASFI clinical score (0-10) & $3.04 \pm 2.0$ & $5.62 \pm 2.3$ & $\mathrm{p}=0.016^{*}, \mathrm{t}=2.655$ \\
MDHAQ function (0-10) & $2.51 \pm 2.7$ & $2.33 \pm 1.46$ & $\mathrm{p}=0.788, \mathrm{t}=0.273$ \\
Pain VAS (0-10) & $4.71 \pm 3.5$ & $5.50 \pm 2.37$ & $\mathrm{p}=0.571, \mathrm{t}=0.578$ \\
Pt-global VAS (0-10) & $4.45 \pm 2.4$ & $13.29 \pm 5.3$ & $\mathrm{p}=0.319, \mathrm{t}=1.024$ \\
MDHAQ-Rapid-3 (0-30) & $11.67 \pm 7.8$ & $\mathrm{p}=0.610, \mathrm{t}=0.518$ \\
\hline
\end{tabular}

BASDAI: Bath Ankylosing Spondylitis Disease Activity Index; BASFI: Bath Ankylosing Spondylitis Functional Index; MDHAQ: Multi-Dimensional Health Assessment Questionnaire; MDHAQ-RAPID-3: Multi-Dimensional Health Assessment Questionnaire-Routine Assessment of Patient Index Data; Pt-global: Patient global assessment. 
The clinical criteria of the modified New York Classification for AS include low back pain and stiffness that improves with exercise but not relieved by rest ${ }^{[15]}$. In the absence of a structured interview, this symptomatology may be misinterpreted as the primary feature of RLS, which is the relief of sensory symptoms by movement. The risk here is the overestimation of RLS prevalence among patients with AS. In our study, this condition was taken into consideration while evaluating the patients.

In our study, RLS was found in 11 (55\%) patients with AS. The clinical severity of RLS was 21.0 \pm 7.91 (severe). The sleep quality was poorer (PSQI score $=8.36 \pm 3.59$ ) in the RLS group than in the non-RLS group.

Sleep dysfunction is very common in patients with RLS and is frequently attributed to their leg symptoms. It is noteworthy that approximately nine of every 10 patients with RLS reported at least one sleep-related symptom. RLS often has a profound impact on sleep and the quality of life [22]

Da Costa et al. ${ }^{[23]}$ used PSQI for evaluating the sleep quality in patients with spondyloarthropathy and found that $68.8 \%$ of patients were poor sleepers (global PSQI score $>5$ ). Addressing sleep problems in AS may have a synergistic effect on pain, functional status, and overall quality of life. In another study which examined sleep quality in patients with AS, the total amount of sleep was significantly lesser in patients with AS than in controls, mainly due to several periods of wakefulness during the night ${ }^{[24]}$. In patients with AS, pain was correlated with difficulty in sleep initiation and poor sleep quality, whereas stiffness was correlated with difficulty in waking and feeling tired and clumsy in the morning ${ }^{[25]}$.

We speculated that RLS was a result of inflammation and/ or autoimmunity in patients with AS, because there was no metabolic abnormality or low serum ferritin levels in these patients.

RLS was found in a less proportion of patients with permanent disability (patients with low scores in the modified Schober test and those with high scores in BASFI). Physical function was measured using BASFI and remained impaired owing to the structural damage to the spine ${ }^{[26]}$. We observed that symptoms, especially pain and stiffness, due to RLS could be prominently seen in patients with permanent disability. Therefore, the symptoms associated with RLS could not be enunciated by patients with AS.

The medication taken by patients with AS and by controls represents an issue. For instance, opiates and calcium channel ligands, such as pregabalin and gabapentin, could be administered for residual pain in AS when nonsteroidal anti-inflammatory drugs are contraindicated. These drug classes could mask RLS symptoms and introduce a confusion bias. This condition was taken into consideration while evaluating the patient and control groups in our study.

The present study had several limitations. The study was performed with a small patient group in which most patients were male. This problem can be evaluated by studies with more number of patients and with no gender differences. Furthermore, we did not evaluate FM, which is more frequent in women and is now considered to be the most common cause of generalized musculoskeletal pain in women aged $20-55$ years ${ }^{[27]}$. It is frequently associated with other rheumatic diseases, such as RA and systemic lupus erythematosus (SLE) ${ }^{[28]}$. Additionally, it is known that a higher prevalence of RLS is observed in patients with FM ${ }^{[29]}$. However, there are few reports on the prevalence of FM in patients with AS. In a study, only female patients with AS were evaluated, and FM was found to be present in $50 \%$ of female patients with AS ${ }^{[30]}$. In our study, only a quarter of patients were female, and FM was not evaluated.

The pathophysiology of RLS is not clear; however, pharmacological evidence indicates that the disorder is related to dopaminergic dysfunction in the central nervous system. Certain dopamine antagonists exacerbate the symptoms of RLS, while dopamine agonists relieve them ${ }^{[3,6,9,11]}$. We could not evaluate the response to dopaminergic treatment for RLS.

In conclusion, we found that RLS and poor sleep quality were highly rated in patients with AS. We also determined an opposite correlation between spinal damage and RLS. We emphasized that it is important to determine the existence of RLS and poor sleep quality in patients with AS. Therefore, further examination by applying the diagnostic criteria for RLS and PSQI in patients with AS will be useful. Further studies are needed to evaluate the presence of RLS in more number of patients with AS.

Peer-review: Externally peer-reviewed.

Authorship Contributions: Concept: G.A.Y.; Design: G.A.Y.; Data Collection or Processing: D.K.; Analysis or Interpretation: H.T.; Literature Search: G.A.Y; Writing: G.A.Y.

Conflict of Interest: None declared.

Financial Disclosure: The authors declared that this study received no financial support.

\section{References}

1. Khan MA. Update on spondyloarthropathies. Ann Intern Med 2002;136:896-907. [CrossRef]

2. Günaydin R, Göksel Karatepe A, Ceşmeli N, Kaya T. Fatigue in 
patients with ankylosing spondylitis: relationships with disease-specific variables, depression, and sleep disturbance. Clin Rheumatol 2009;28:1045-51. [CrossRef]

3. Hening WA, Caivano CK. Restless legs syndrome: a common disorder in patients with rheumatologic conditions. Semin Arthritis Rheum 2008;38:55-62. [CrossRef]

4. Kushida CA. Clinical presentation, diagnosis, and quality of life issues in restless legs syndrome. Am J Med 2007;120:S4-12.

5. Zucconi M, Ferini-Strambi L. Epidemiology and clinical findings of restless legs syndrome. Sleep Med 2004;5:293-9.

6. Stiasny-Kolster K, Trenkwalder C, Fogel W, Greulich W, Hahne $M$, Lachenmayer $L$, et al. Restless legs syndrome--new insights into clinical characteristics, pathophysiology, and treatment options. J Neurol 2004;251 Suppl 6:Vl/39-43.

7. Ondo W, Tan EK, Mansoor J. Rheumatologic serologies in secondary restless legs syndrome. Mov Disord 2000;15:321-3.

8. Desautels A, Turecki G, Montplaisir J, Xiong L, Walters AS, Ehrenberg $B L$, et al. Restless legs syndrome: confirmation of linkage to chromosome $12 q$, genetic heterogeneity, and evidence of complexity. Arch Neurol 2005;62:591-6. [CrossRef]

9. Gamaldo CE, Earley CJ. Restless legs syndrome: a clinical update. Chest 2006;130:1596-604. [CrossRef]

10. Salih AM, Gray RE, Mills KR, Webley M. A clinical, serological and neurophysiological study of restless legs syndrome in rheumatoid arthritis. Br J Rheumatol 1994;33:60-3. [CrossRef]

11. Zucconi M, Manconi M, Ferini Strambi L. Aetiopathogenesis of restless legs syndrome. Neurol Sci 2007;28 Suppl 1:S47-52.

12. O'Keeffe ST. Iron deficiency with normal ferritin levels in restless legs syndrome. Sleep Med 2005;6:281-2. [CrossRef]

13. Allen RP, Picchietti D, Hening WA, Trenkwalder C, Walters AS, Montplaisi J; Restless Legs Syndrome Diagnosis and Epidemiology workshop at the National Institutes of Health; International Restless Legs Syndrome Study Group. Restless legs syndrome: diagnostic criteria, special considerations, and epidemiology. A report from the restless legs syndrome diagnosis and epidemiology workshop at the National Institutes of Health. Sleep Med 2003;4:101-19. [CrossRef]

14. Walters AS, LeBrocq C, Dhar A, Hening W, Rosen R, Allen RP, et al. Validation of the International Restless Legs Syndrome Study Group rating scale for restless legs syndrome. Sleep Med 2003;4:121-32. [CrossRef]

15. van der Linden S, Valkenburg HA, Cats A. Evaluation of diagnostic criteria for ankylosing spondylitis. A proposal for modification of the New York criteria. Arthritis Rheum 1984;27:361-8.

16. Garrett S, Jenkinson T, Kennedy LG, Whitelock H, Gaisford P, Calin A. A new approach to defining disease status in ankylosing spondylitis: the Bath Ankylosing Spondylitis Disease Activity Index. J Rheumatol 1994;21:2286-91.

17. Calin A, Garrett S, Whitelock H, Kennedy LG, O'Hea J, Mallorie
P, et al. A new approach to defining functional ability in ankylosing spondylitis: the development of the Bath Ankylosing Spondylitis Functional Index. J Rheumatol 1994;21:2281-5.

18. Pincus T, Bergman MJ, Yazici Y. RAPID3-an index of physical function, pain, and global status as "vital signs" to improve care for people with chronic rheumatic diseases. Bull NYU Hosp Jt Dis 2009;67:211-25.

19. Buysse DJ, Reynolds CF 3rd, Monk TH, Berman SR, Kupfer DJ. The Pittsburgh Sleep Quality Index: a new instrument for psychiatric practice and research. Psychiatry Res 1989;28:193-213.

20. Johns MW. A new method for measuring daytime sleepiness: the Epworth sleepiness scale. Sleep 1991;14:540-5. [CrossRef]

21. Krupp LB, LaRocca NG, Muir-Nash J, Steinberg AD. The fatigue severity scale. Application to patients with multiple sclerosis and systemic lupus erythematosus. Arch Neurol 1989;46:1121-3. [CrossRef]

22. Taylor-Gjevre RM, Gjevre JA, Skomro R, Nair B. Restless legs syndrome in a rheumatoid arthritis patient cohort. J Clin Rheumatol 2009;15:12-5. [CrossRef]

23. Da Costa D, Zummer M, Fitzcharles MA. Determinants of sleep problems in patients with spondyloarthropathy. Musculoskeletal Care 2009;7:143-61. [CrossRef]

24. Hultgren S, Broman JE, Gudbjörnsson B, Hetta J, Lindqvist U. Sleep disturbances in outpatients with ankylosing spondylitisa questionnaire study with gender implications. Scand J Rheumatol 2000;29:365-9. [CrossRef]

25. Jamieson AH, Alford CA, Bird HA, Hindmarch I, Wright V. The effect of sleep and nocturnal movement on stiffness, pain, and psychomotor performance in ankylosing spondylitis. Clin Exp Rheumatol 1995;13:73-8.

26. Landewé $R$, Dougados $M$, Mielants $H$, van der Tempel $H$, van der Heijde D. Physical function in ankylosing spondylitis is independently determined by both disease activity and radiographic damage of the spine. Ann Rheum Dis 2009;68:863-7.

27. Rustøen T, Wahl AK, Hanestad BR, Lerdal A, Paul S, Miaskowski $C$. Age and the experience of chronic pain: differences in health and quality of life among younger, middle-aged, and older adults. Clin J Pain 2005;21:513-23. [CrossRef]

28. Wolfe F, Michaud K. Severe rheumatoid arthritis (RA), worse outcomes, comorbid illness, and sociodemographic disadvantage characterize ra patients with fibromyalgia. J Rheumatol 2004;31:695-700.

29. Viola-Saltzman M, Watson NF, Bogart A, Goldberg J, Buchwald D. High prevalence of restless legs syndrome among patients with fibromyalgia: a controlled cross-sectional study. J Clin Sleep Med 2010;6:423-7.

30. Aloush V, Ablin JN, Reitblat T, Caspi D, Elkayam O. Fibromyalgia in women with ankylosing spondylitis. Rheumatol Int 2007;27:865-8. [CrossRef] 